\title{
Erratum: Numerical Study of a Dual Representation of the Integer Quantum Hall Transition [Phys. Rev. Lett. 126, 056802 (2021)]
}

Kevin S. Huang, S. Raghu, and Prashant Kumar॰

Q (Received 9 July 2021; published 6 August 2021)

DOI: 10.1103/PhysRevLett.127.069903

The following sentence made in our Letter: "Likewise, we also find evidence for corrections to the proposed parabolic form [50-53] since $\gamma_{1} \neq 0$ " below Eq. (8) cannot be justified based on the numerics reported in the Letter, since the error bars of $\gamma_{1}$ were found to be equal or bigger than its magnitude. Thus, our results are consistent with the multifractal spectrum being parabolic. This correction does not alter any conclusions of the Letter. 